\title{
Efectividad del taller "superando disciplinas" en el rendimiento académico de alumnos del colegio Víctor Alberto Peña Neyra, Tumbes 2020
}

\author{
Rosa Vanessa, Becerra Ortiz \\ rbecerrao@ucvvirtual.edu.pe \\ https://orcid.org/0000-0001-5049-9805 \\ Universidad César Vallejo \\ Dulio Oseda Gago \\ dosedag@ucvvirtual.edu.pe \\ https://orcid.org/0000-0002-3136-6094 \\ Universidad César Vallejo
}

\section{RESUMEN}

Muchos son los estudiantes en el mundo, que se encuentran inmersos dentro de un ciclo vicioso, donde prevalece el bajo rendimiento académico y niveles elevados de desmotivación, es así que la finalidad principal de la investigación fué determinar la efectividad del taller "Superando disciplinas" en el rendimiento académico de alumnos del colegio Víctor Alberto Peña Neyra, Tumbes 2020, tratándose de un estudio con enfoque cuantitativo - pre experimental, cuyo universo lo conformaron 137 estudiantes de nivel secundario y la muestra representativa 29 estudiantes, a quienes se les aplico [antes y después de recibir el taller -superando Disciplinas-] un cuestionario estructurado, compuesto de 20 preguntas de propia autoría, sometido al proceso de validación y prueba de confiabilidad respectiva. Resultados: Durante el pre test el rendimiento académico en la mayoría de estudiantes 79,3\% estaba en INICIO, mientras que después de recibir el taller este mejoró notablemente en el $93.1 \%$ de estudiantes, quienes alcanzaron el LOGRO ESPERADO y en un 6,9\% de los alumnos aún permanecía en PROCESO, finalmente al realizar el análisis de los resultados obtenidos durante el pre y post test; y aplicar la prueba estadística Wilcoxon, se obtuvo un nivel de sig. bilateral de $p=0,000$ (donde $\mathrm{p}<0,05$ ), infiriendo que los puntajes antes y después de la aplicación del taller Superando Disciplinas son significativamente diferentes, por lo tanto se acepta la hipótesis alterna y se afirma que el taller Superando Disciplinas resultó ser efectivo para mejorar el rendimiento académico de los alumnos del colegio Víctor Alberto Peña Neyra - Tumbes 2020.

Palabras clave: efectividad; taller superando disciplinas; rendimiento académico; estudiante; estrategias textuales; aprendizaje; comprensión; lectura; nivel literal e inferencial. 


\title{
Effectiveness of the workshop "overcoming disciplines" in the academic performance of students of the Víctor Alberto Peña Neyra school, Tumbes 2020
}

\begin{abstract}
Many are the students in the world, who are immersed in a vicious cycle, where low academic performance and high levels of demotivation prevail, so the main purpose of the research was to determine the effectiveness of the workshop "Overcoming disciplines" in the academic performance of students from the Víctor Alberto Peña Neyra school, Tumbes 2020, being a study with a quantitative approach - pre-experimental, whose universe was made up of 137 high school students and the representative sample 29 students, to whom [before and after receiving the workshop -overcoming Disciplines] a structured questionnaire, made up of 20 questions of their own authorship, submitted to the validation process and respective reliability test. Results: During the pre-test, the academic performance in the majority of students was $79.3 \%$ in START, while after receiving the workshop, it improved notably in $93.1 \%$ of students, who achieved EXPECTED ACHIEVEMENT and in $6.9 \%$ of the students still remained in PROCESS, finally when carrying out the analysis of the results obtained during the pre and post test; and applying the Wilcoxon statistical test, a level of sig was obtained. bilateral of $p=$ 0.000 (where $\mathrm{p}<0.05$ ), inferring that the scores before and after the application of the Overcoming Disciplines workshop are significantly different, therefore the alternative hypothesis is accepted and it is stated that the Overcoming Disciplines workshop turned out to be effective to improve the academic performance of the students of the Víctor Alberto Peña Neyra - Tumbes 2020 school.
\end{abstract}

Keywords: effectiveness; overcoming disciplines workshop; academic performance; student; textual strategies; learning; comprehension; reading; literal and inferential level.

Artículo recibido:.10 Agosto. 2021 Aceptado para publicación: 07. Setiembre. 2021 Correspondencia: rbecerrao@ucvvirtual.edu.pe Conflictos de Interés: Ninguna que declarar 


\section{INTRODUCCIÓN}

En la actualidad la educación de tipo básica se destina al favorecimiento íntegro del proceso de desarrollo del educando, a propiciar el descubrimiento de la potencialidad e incentivar el desarrollo de capacidad, conocimiento, actitud, asimismo de valores claves, que el individuo, debe tener para una actuación adecuada y eficaz en los diferentes contextos sociales, es así, que, todos los países realizan acciones que apuntan a la mejora del rendimiento de los educandos, basándose en la implementación de políticas públicas efectivas, pues países cuya economía es tan variante como Brasil, México, Túnez, Turquía, Italia, Polonia, Portugal, Rusia y Alemania, lograron reducir el porcentaje de estudiantes con bajo rendimiento académico. (MINISTERIO DE EDUCACION, 2017). A nivel de Latinoamérica son Perú, Colombia, Brasil y Argentina, los países que están entre las diez naciones con altos índices de rendimiento académico bajo en matemáticas, lectura y ciencia, datos obtenidos de una investigación desarrollada por la Organización para la Cooperación y el Desarrollo Económicos (OCDE), en 64 naciones y publicada en febrero 2016.

La república del Perú tiene un alto porcentaje de alumnos de 15 años que no lograron superar el nivel básico de lectura (60 por ciento) ciencia (68,5 por ciento), además ocupa el lugar número dos de bajo rendimiento en matemáticas (74,6 por ciento), encontrándose por debajo de Indonesia, durante el año 2015, el 49.8\% de estudiantes de $2^{\circ}$ grado de primaria evaluados por el MINEDU en la Evaluación Censal de Estudiantes (ECE) obtuvieron niveles satisfactorios en comprensión lectora y $26.6 \%$ en matemática, esto de alguna manera, representa un notable progreso si se compara con las cifras alcanzadas durante el año 2009, donde los valores eran inferiores (MINISTERIO DE EDUCACION, 2017).

En la región Tumbes, según cifras obtenidas de la evaluación censal de estudiantes 2018, se desprende que sólo 22 de cada 100 estudiantes de cuarto grado de primaria en la Región Tumbes logran un nivel satisfactorio en comprensión, asimismo sólo 17 de cada 100 estudiantes alcanzan un nivel satisfactorio en Matemáticas. (Mesa de concertacion para la lucha contra la pobreza Tumbes., 2019)

La institución educativa $\mathrm{N}^{\circ} 118$ Víctor Alberto Peña Neyra, no escapa a estas evaluaciones con resultados poco deseables para el nivel secundaria, nuestros estudiantes proceden de hogares disfuncionales, con actitudes negativas, carentes de aspiraciones y 
motivaciones para culminar con su educación, ya que muchas veces los padres poseen un bajo nivel cultural y demás circunstancias que influyen en el comportamiento y sentimientos de los estudiantes afectando directa o indirectamente el rendimiento académico.

Yerco (2017) indica que en el rendimiento académico interfieren elementos específicos donde está inmerso básicamente el contexto familiar inmediato, sociocultural y entorno educativo, etc., relacionados básicamente a los resultados obtenidos en los educandos, estos pueden expresarse y manifestarse en un contexto físico, el cual se afianza, determina y caracteriza durante la línea de tiempo, asimismo, se indica que los problemas asociados a los comportamientos, son básicamente un desafío dentro de la escuela y el sistema mismo, pues se convierten en dificultades para aquellos profesionales con conexión directa con los niños y jóvenes. Dichas problemáticas están presenten en mayor incidencia en aquellos lugares con mayor vulnerabilidad, llámense profesores, padres y apoderados, quienes suelen problemas relacionados al trato hacia el alumnado con grandes problemas de convivencia y desempeño relacionado con el adecuado proceso de aprendizaje.

Ante la situación antes descrita la autora ha creído conveniente e imperativa la necesidad de abordar esta problemática mediante la presente investigación partiendo de la siguiente pregunta: ¿Cuál es la efectividad del taller Superando disciplinas en el rendimiento académico de alumnos del colegio Víctor Alberto Peña Neyra, Tumbes 2020

El estudio que la autora llevó a cabo resulta ser de gran relevancia social, sustentándose, que es precisamente la educación el pilar fundamental, alineado a los objetivos de desarrollo sostenible, asimismo garantiza el trato equitativo dentro de la sociedad por tratarse de uno de los determinantes fundamentales que mide el grado de desarrollo de una nación. Es necesario que desde los distintos niveles de gobierno nacional, local y regional se generen las mejores condiciones garantizadoras de la educación de calidad e igualitaria, asimismo que las autoridades competentes como las direcciones regionales de educación evalúen constantemente las estrategias educativas que apunten a logros de aprendizajes positivos en los educandos.

En relación al aporte teórico, a través de la investigación se podrá enriquecer el acervo cognitivo, ya existente respecto a las estrategias, técnicas y/o acciones que favorecen y mejoran el rendimiento académico en el alumnado, sobremanera en etapas tan trascendentales como lo es la adolescencia. Mientras que el aporte practico se sustenta, 
que, a partir de los resultados obtenidos, serán las autoridades competentes las indicadas de implementar dichas estrategias a nivel de la comunidad educativa, propiciando el fortalecimiento y la mejorara del rendimiento académico en estudiantes de la región de Tumbes, asimismo generarán las condiciones para facilitar el aprendizaje.

En relación al aporte metodológico se respalda en el instrumento mismo, el cual gozó de confiabilidad y validez, garantizando su utilidad para posteriores investigaciones relacionadas con la problemática que se estudió en esta investigación.

Para el estudio se ha planteado como:

\section{OBJETIVO GENERAL}

- Determinar la efectividad del taller superando disciplinas en el rendimiento académico de alumnos del colegio Víctor Alberto Peña Neyra, Tumbes 2020.

\section{Como objetivos específicos se plantearon}

\section{Objetivo específico 1}

- Conocer la efectividad del taller superando disciplinas para mejorar la aplicación de la disciplina en la vida cotidiana en los alumnos del colegio Víctor Alberto Peña Neyra, Tumbes 2020.

\section{Objetivo específico 2}

- Conocer la efectividad del taller superando disciplinas para mejorar el manejo de información adecuada sobre un tema específico en los alumnos del colegio Víctor Alberto Peña Neyra, Tumbes 2020.

\section{HIPÓTESIS GENERAL}

- El taller superando disciplinas es efectivo para mejorar el rendimiento académico en los alumnos del colegio Víctor Alberto Peña Neyra, Tumbes 2020.

\section{Como hipótesis específicas se plantearon:}

\section{Hipótesis específica 1}

- El taller superando disciplinas es efectivo para mejorar la aplicación de disciplina en la vida cotidiana en los alumnos del colegio Víctor Alberto Peña Neyra, Tumbes 2020.

\section{Hipótesis específica 2}

- El taller superando disciplinas es efectivo para mejorar el manejo de información adecuada sobre un tema específico en los alumnos del colegio Víctor Alberto Peña Neyra, Tumbes 2020.

\section{ESTRATEGIAS METODOLÓGICAS O MATERIALES Y MÉTODOS}




\section{Tipo y diseño de estudio}

La investigación tuvo un enfoque metodológico cuantitativo, de tipo descriptivo explicativo, en cuanto al diseño, fue pre experimental, en la medida en que se aplicó, un pre y post test, en un solo grupo muestral, al cual se le aplica una prueba previa al estímulo o tratamiento experimental, después se le administra el tratamiento y finalmente se aplica la prueba posterior al estímulo (Hernández, Fernández y Batista, 2010: 136).

\section{La población}

Estuvo conformado por 137 alumnos matriculados en el nivel secundario de la Institución Educativa $\mathrm{N}^{\circ} 118$ " Víctor Alberto Peña Neyra - Tumbes, durante el periodo educativo anual 2020.

\section{Muestra}

Estuvo conformada por 29 estudiantes, matriculados en el segundo grado de secundaria de la IE N¹18 - Víctor Alberto Peña Neyra.

\section{Técnica e Instrumento}

Para la ejecución del estudio se utilizó la encuesta como técnica [pre y post Test], cuya finalidad básicamente fue obtener información respecto a la efectividad del taller Superando Disciplinas - en el nivel de rendimiento académico, del grupo de adolescentes participes del estudio. Respecto al instrumento la autora elaboró un cuestionario pre codificado, auto aplicable, de naturaleza anónima, cabe señalar que este fue sometido al respectivo proceso de confiabilidad y validación. Asimismo, este cuestionario estuvo compuesto de 20 preguntas, subdividido a su vez en dos dimensiones: Actitudinal [1 -10 ítems] y cognitivo [11 -20 ítems], el cual fue llenado con la presencia de la autora quien pudo resolver las distintas dudas que puedan haber surgido durante el llenado.

\section{Procedimientos}

Se pudo recoger información selecta, antes de la aplicación del taller y después de la aplicación, asimismo luego, se realizó la comparación del comportamiento de las variables en ambos momentos. El taller - Superando Disciplinas - se logró desarrollar en 5 sesiones de manera virtual a través de la plataforma ZOOM, en cada sesión se abordaron tres temáticas especificas relacionadas a los objetivos específicos para reconocer actitudes 
que impiden y fortalecer el aprendizaje, con la adquisición del autocontrol y el uso de habilidades sociales.

\section{RESULTADOS Y DISCUSIÓN}

A continuación, se presenta los resultados:

Tabla 1 Tabla de frecuencia según nivel de efectividad del taller superando disciplinas en el rendimiento académico en alumnos del colegio Víctor Alberto Peña Neyra, Tumbes 2020

\begin{tabular}{l|ccccc}
\hline \multirow{1}{*}{ Niveles } & \multicolumn{4}{c}{ Rendimiento académico } \\
& Pr & \% & fi & Pos test \\
\hline En inicio & 23 & 79,3 & 0 & 0,0 \\
En proceso & 5 & 17,2 & 2 & 6,9 \\
Logrado & 1 & 3,4 & 27 & 93,1 \\
\cline { 1 - 5 } \multicolumn{1}{c}{ Total } & $\mathbf{2 9}$ & $\mathbf{1 0 0 , 0}$ & $\mathbf{2 9}$ & $\mathbf{1 0 0 , 0}$ \\
\hline
\end{tabular}

\section{Interpretación:}

De acuerdo a la tabla 1; muestran los resultados globales de la investigación sobre la efectividad del taller superando disciplina en el rendimiento académico, reportado en estudiantes de Educación Básica Regular del colegio Víctor Alberto Peña Neyra, de la ciudad de Tumbes en el año 2020; en ellos se visualiza que antes de la aplicación del experimento (pre test) el 79,3\% de los estudiantes, en rendimiento académico se encontraban en inicio, solo el 3,4\% se encontraban en el nivel logrado; mientras que, luego de la aplicación del experimento (pos test) el 93,1\% de los estudiantes participantes en la investigación se ubicaron en el nivel logrado, el 6,9\% en proceso y ningún estudiante están en inicio, con lo que se puede afirmar que el experimento trae buenos resultados, esta afirmación se verificará o desmentirá en la contrastación o prueba de hipótesis.

Tabla 2. Tabla de frecuencia de la primera dimensión aplicación de la disciplina en alumnos del colegio Víctor Alberto Peña Neyra, Tumbes 2020.

\begin{tabular}{l|cccc}
\hline \multirow{2}{*}{ Niveles } & \multicolumn{3}{c}{ Aplicación de disciplina en la vida cotidiana } \\
& \multicolumn{2}{c}{ Pre test } & \multicolumn{3}{c}{ Pos test } \\
\hline Malo & fi & \% & fi & \% \\
\hline Indiferente & 23 & 79,3 & 0 & 0,0 \\
& 5 & 17,2 & 3 & 10,3
\end{tabular}


$\begin{array}{lllll}\text { Bueno } & 1 & 3,4 & 26 & 89,7\end{array}$

\begin{tabular}{|l|l|l|l|l|}
\hline Total & 29 & 100,0 & 29 & 100,0 \\
\hline
\end{tabular}

\section{Interpretación:}

Los resultados que se muestran en la tabla 2 sobre la aplicación de la disciplina, como parte de la variable dependiente rendimiento académico, en el pre test el 79,3\% de los estudiantes manifiesta una mala actitud ante la aplicación de la disciplina en estudiantes del colegio Víctor Alberto Peña Neyra, al 17,2\% le es indiferente y solo al 3,4\% muestran buena actitud; por otra parte, en el pos test es decir luego de la aplicación del experimento, un grupo representativo del $89,7 \%$ de estudiantes tienen un nivel de actitud alto en cuanto a la aplicación de la disciplina, mientras que al 10,3\% de participantes en la investigación le es indiferente la aplicación de la disciplina; estos resultados muestran que el programa "efectividad del taller superando disciplinas" tiene efectos favorables con respecto a la aplicación de la disciplina, resultados que serán corroborados con la a

Tabla de frecuencia de la segunda dimensión manejo de información adecuada.

Tabla 3. Tabla de frecuencia de la segunda dimensión manejo de información adecuada sobre un tema específico en alumnos del colegio Víctor Alberto Peña Neyra, Tumbes 2020.

\begin{tabular}{|c|c|c|c|c|}
\hline \multirow{3}{*}{ Niveles } & \multicolumn{4}{|c|}{ Manejo de información adecuada sobre un tema específico } \\
\hline & \multicolumn{2}{|c|}{ Pre test } & \multicolumn{2}{|c|}{ Pos test } \\
\hline & fi & $\%$ & $\mathbf{f i}$ & $\%$ \\
\hline En inicio & 28 & 96,6 & 0 & 0,0 \\
\hline En proceso & 1 & 3,4 & 2 & 6,9 \\
\hline Logrado & 0 & 0,0 & 27 & 93,1 \\
\hline Total & 29 & 100,0 & 29 & 100,0 \\
\hline
\end{tabular}

\section{Interpretación:}

Los resultados que se muestran en la tabla 10 sobre el manejo de información adecuada sobre un tema específico, como parte de la variable dependiente rendimiento académico, dan cuenta que en pre test el 96,6\% de estudiantes del colegio Víctor Alberto Peña Neyra de la ciudad de Tumbes, se encuentran en la etapa de Inicio en el manejo de información adecuada sobre un tema específico y solo el 3,4\% han logrado superar esta etapa; por otra parte, luego del tratamiento de la variable independiente "efectividad del taller superando disciplinas" el 93,1\% han logrado manejar adecuadamente un tema específico, ninguno 
está en la etapa en inicio como en el pre test y el 6,9\% se encuentra en proceso de lograr manejar adecuadamente $\mathrm{u}$ tema específico referido al rendimiento académico.

\section{Planteamiento de la hipótesis:}

Hipótesis nula: Ho: El taller superando disciplinas no es efectivo para mejorar el rendimiento académico en los alumnos del colegio Víctor Alberto Peña Neyra, Tumbes 2020.

Hipótesis alterna: Ha: El taller superando disciplinas es efectivo para mejorar el rendimiento académico en los alumnos del colegio Víctor Alberto Peña Neyra, Tumbes 2020.

Nivel de significancia o riesgo: $\alpha=0,05=5 \%$.

Decisión estadística: De esta manera; al presentar un p-valor: $\mathrm{p}<\alpha$, se tiene como consecuencia el rechazo de la hipótesis nula (Ho) y la aceptación de la hipótesis alterna (Ha).

Conclusión estadística: Se concluye ser efectivo utilizando el estadístico para muestras relacionadas Wilcoxon donde $\mathrm{p}<0.05$ por lo que, el taller superando disciplinas es efectivo para mejorar el rendimiento académico en los alumnos del colegio Víctor Alberto Peña Neyra, Tumbes 2020.

\section{Interpretación:}

En la tabla estadísticos de prueba, el valor de $\mathrm{z}=-4,726$ siendo $\mathrm{z}<-1,96$ este valor indica que $\mathrm{z}$ se encuentra dentro del valor permitido y además el valor de la sig. asintótica (bilateral) o "p valor" es 0,000 siendo este menor que $\alpha=0,05$ por lo tanto, $p<\alpha$ y de acuerdo a la regla de decisión, se rechaza la hipótesis nula y se acepta la hipótesis alternativa, concluyéndose que "el taller superando disciplinas es efectivo para mejorar el rendimiento académico en los alumnos del colegio Víctor Alberto Peña Neyra, Tumbes 2020”.

\section{Discusión}

Después de obtener cada hallazgo es necesario iniciar este apartado mencionando que la educación es el pilar o columna vertebral del desarrollo en una nación, promover cultura es la mejor herramienta para enfrentar problemáticas de índole estructural presente en nuestras comunidades, razón por la cual el docente y directivos dentro del escenario educativo deben estar prestos a adoptar nuevas herramientas o estrategias, que apunten a la mejora y optimización de condiciones para un desarrollo adecuado del proceso 
educativo, rendimiento académico y aprendizaje, al respecto en la tabla 7 y 10, se puede evidenciar que la propuesta de taller "Superando disciplinas" resulto ser de gran efectividad Wilcoxon $\mathrm{p}=0,000$, $(\mathrm{p}<0.05)$, para la mejora del rendimiento académico de alumnos del colegio Víctor Alberto Peña Neyra, Tumbes 2020, puesto que se sustenta con los datos descriptivos obtenidos en la tabla 4 la cual indica respecto al nivel de rendimiento académico, que permanecía en inicio en el 79,3\% y solo un $17,2 \%$ se encontraba en proceso, antes de la aplicación del taller (Pre test).

Estos datos resultan similares a los obtenidos por Osorio \& Castiblanco (2019), quienes llegan a deducir que el programa de lecto escritura, aplicado por ellos, [b-learning] contribuyó a mejorar la tasa de retención estudiantil, para finalmente concluir que la implementación de b-learning mejora tanto el porcentaje de aprobación del curso, como la retención estudiantil.

Asimismo, Almendárez J.; Carrasco [2017] concluyen que la disciplina positiva se constituye en una condición idónea para alcanzar metas y propósitos educativos, Para Figueroa \& Chumpitaz, (2018) su propuesta de Blog educativo tuvo un efecto positivo en el rendimiento académico en matemática en un grupo de estudiantes del segundo año de secundaria.

Contrariamente Escalante (2018), no logró encontrar relación directa significativa estadísticamente $(r=.021, .806<0.05)$ entre bienestar psicológico y rendimiento académico en los estudiantes de primer año de formación inicial docente del Instituto Pedagógico Nacional Monterrico, sugiriendo se deben hacer mayores investigaciones.

Woolfolk (1995).considera que el rendimiento está influenciado por factores internos como externos, es el autocontrol quien contempla el comportamiento que se dirige a la obtención de una recompensa que puede ser física o emocional, dicho postulado se ajusta a lo encontrado durante el post test, básicamente después de aplicado el taller superando disciplinas, la autora evidenció que el nivel de rendimiento académico mejoró notablemente, un $93.1 \%$ de los estudiantes obtuvieron un nivel de logro esperado del rendimiento académico mientras que el 6.9\% de estudiantes del colegio Víctor Alberto Peña Neyra, se encontraban en PROCESO [tabla 4 y figura 2].

De igual forma, Figueroa \& Chumpitaz, (2018), indican que al aplicar el post test el rendimiento académico de los estudiantes del grupo experimental fue significativamente mayor que el rendimiento académico de los estudiantes del grupo de control, un panorama 
casi similar encontró Ocampos (2018) quien concluye que previa a la intervención y aplicación del pre test un Logro previsto de $81 \%$ y un Logro en proceso de 19\%; asimismo en el post test se presenta un Logro destacado de $94 \%$ y un Logro previsto de $6 \%$.

Otro aporte fundamental del estudio se enmarca en los comportamientos de los alumnos participes de estudio antes de la intervención predominaban comportamientos y actitudes NEGATIVAS [79,3\%], frente al rendimiento académico, esta situación mejoró post taller, puesto que prevalecen BUENAS actitudes en un $89,7 \%$ de estudiantes, dicho resultado es interesante porque denota al aprendizaje no solo como un proceso cognitivo sino además que este es regulado y afectado por otros factores, en relación a este punto traeré a acotación El modelo ecológico de aprendizaje, este es enriquecedor, puesto que parte del trabajo en equipo, resaltando el liderazgo, tratando de identificar el papel desempeñado por el estudiante, visualizando quien necesita apoyo y quien puede ofrecerlo, produciendo un trabajo colaborativo pudiendo establecer vínculos sociales y el desarrollo de reglas para una mejor convivencia dentro del circulo escolar.

Al respecto Almendárez, J.; Carrasco, sostienen, que la disciplina positiva se constituye en una condición idónea para alcanzar metas y propósitos educativos, puesto que el aprendizaje se desarrolla de forma óptima y por tanto con espacios y oportunidades formativas y propicias., Fajardo, F; Maestre, M; et al (2017) por su parte indican que escuela de calidad es aquella que sabe dar respuesta a las necesidades específicas de todos y cada uno de los alumnos, que es capaz de potenciar sus capacidades de manera individualizada.

Mientras que Concepcion (2017) propone sistematizar las estrategias de acompañamiento para brindarles herramientas a los padres de familia para su formación y así de una manera más responsable ellos contribuyan en la mejora del rendimiento escolar, es interesante el aporte por este autor, anteriormente hablábamos de factores externos, precisamente es imperativo que la familia se involucre más en el proceso educativo formativo de sus hijos, tal es así que si se presentaran más condiciones favorecedoras para la educación es mejor y el impacto positivo que genera en el educando y educador.

Como se puede observar en el estudio, es necesario la búsqueda de herramientas o intervenciones claves, sostenibles para poder replicarlas y desarrollar las potencialidades cognitivas y actitudinales de los estudiantes, estas se alinearán a los requerimientos específicos e individualizados de cada estudiante. 


\section{CONCLUSIÓN O CONSIDERACIONES FINALES}

\section{Conclusiones}

- Primera Conclusión: El taller resultó ser efectivo utilizando el estadístico para muestras relacionadas Wilcoxon donde $\mathrm{p}<0.05$ por lo que, el taller superando disciplinas es efectivo para mejorar el rendimiento académico en los alumnos del colegio Víctor Alberto Peña Neyra, Tumbes 2020.

- Segunda Conclusión: El taller resultó ser efectivo para mejorar la disciplina utilizando el estadístico para muestras relacionadas Wilcoxon, donde $\mathrm{p}<0.05$ por lo que, el Taller superando disciplinas es efectivo para mejorar la aplicación de disciplina en la vida cotidiana en los alumnos del colegio Víctor Alberto Peña Neyra, Tumbes 2020.

- Tercera Conclusión: El taller resultó ser efectivo para mejorar la disciplina utilizando el estadístico para muestras relacionadas Wilcoxon, donde $\mathrm{p}<0.05$ por lo que, el Taller superando disciplinas es efectivo para mejorar la aplicación de disciplina en la vida cotidiana en los alumnos del colegio Víctor Alberto Peña Neyra, Tumbes 2020.

- Cuarta conclusión: Los comportamientos de los alumnos participes de estudio antes de la intervención predominaban comportamientos y actitudes INDIFERENTES [51.7\%], frente al rendimiento académico, esta situación mejoró post taller, puesto que prevalecen BUENAS actitudes en un $93.1 \%$ de estudiantes.

\section{Consideraciones finales}

Se logró aplicar con éxito el taller, identificando información relevante, en los diferentes momentos evaluadores pre test y post Test sobre el rendimiento académico [dimensión cognitiva y dimensión actitudinal] en los estudiantes del 2 do grado educación secundaria de la Institución Educativa “Víctor Alberto Peña Neyra”. Las Malvinas Tumbes - 2020, se utilizó dinámica activa con el uso de medios y materiales adecuados, despertando el interés del tema en los estudiantes para reflexionar ente sus conductas y tomar las decisiones oportunas para valorarse como persona y mejorar su proceso de aprendizaje, pues todo taller deja un aprendizaje a desarrollar, y se espera que los estudiantes utilicen diferentes estrategias frente a las dificultades, entre ellas el aprender a convivir de manera armónica y desarrollar el comportamiento pro activo para superar las dificultades y los problemas consecuentes como por ejemplo la deserción y ausentismo escolar. 
La Dirección Regional de Educación Tumbes, debe trabajar de manera conjunta con las unidades de gestión escolar local, para manejar información relevante sobre el proceso de aprendizaje y el impacto en el rendimiento académico, así como también de la disciplina dentro de las escuelas urbano marginal, con el propósito de identificar a los estudiantes infractores, establecer acciones articuladas con los aliados estratégicos del sector, además debe hacer seguimientos, para brindar atención de acuerdo a las realidades y necesidades de cada institución educativa, los directivos deberán ser consientes que el trabajo no solo es de oficina, es relevante el asesoramiento en normas, leyes que hoy están vigentes y respaldan el mantenimiento de una convivencia escolar saludable, siendo este el principal determinante para garantizar en el alumno un clima adecuado para el aprendizaje por ende la mejora o la optimización en su rendimiento académico.

A los integrantes de la Institución Educativa [directivo, docentes entre otros] poner énfasis en la disciplina; no solo importa cuánto se ha alcanzado en conocimientos, sino también la parte de la conducta, considerar los pequeños talleres para estudiantes, docentes y todos lo que laboran en la institución educativa para poner énfasis en este tema y hacer reflexionar sobre las actitudes desde cada ámbito que nos toca desarrollar, manejando estrategias adecuadas de liderazgo, motivar a los docentes a involucrarse con la problemática de la institución y la autoformación para poder solucionar los problemas de indisciplina que dan en la institución, se debe buscar ayuda con los colegas directivos compartir los casos y buscar las estrategias adecuadas para el desarrollo efectivo de las actividades educativas.

Como docente debemos tener apertura a las necesidades de los estudiantes, motivarlos y considerar cada uno de los estilos de aprendizaje que tiene el estudiante para aprender y planificar según sus necesidades; si hacemos nuestro trabajo con amor y empeño, cuando brindamos esa confianza quizá el estudiante se sienta valorado y en algún momento si se encontrara en una situación difícil para decidir, se acordara de lo que le dijo su docente y tomara la decisión correcta. Si ponemos responsabilidad y algo de amor a nuestro trabajo uniendo fuerzas para mejorar de seguro cambiaremos el rumbo que experimenta la sociedad de hoy.

\section{LISTA DE REFERENCIAS}

Adell, m.a. (2006). Estrategias para mejorar el rendimiento académico de los adolescentes. Madrid: pirámide. 
Almendárez, j.; carrasco. Et al (2017) estrategias para el fortalecimiento de la disciplina positiva. De la ciudad de estelí-nicaragua.

Artunduaga, m. (2008). Variables que influyen en el rendimiento académico en la universidad. Recuperado de http://goo.gl/o5r0nx.

Barrios, m., \& frias, m. (2015). Factores que influyen en el desarrollo y rendimiento escolar de los jovenes de bachillerato. Sonora - mexico.

Boeree, gene. S/f. Alfred adler. Personality theories. Último acceso: 10 de noviembre de 2017. Http://www.ship.edu/ cgboeree/adler.html.

Bringas.c, rodríguez, f. Et al (2009). Responsabilidad y comportamiento antisocial del adolescente como factores asociados al rendimiento escolar. Acta colombiana de psicología, 12, 69-76.

Bunge, m. La epistemología o filosofía de la ciencia.

Cascón, i. V. (2000). Análisis de las calificaciones escolares como criterio de rendimiento académico. Recuperadode https://goo.gl/fxpags.

Concepcion, b. (2015). Estrategias de acompañamiento a padres de familia para mejorar el rendimiento escolar de los estudiantes de la ie puruay-centro poblado rio grande cajamarca. Cajamarca.

Contreras, m. A., corbalán, f. Y redondo, j. (2007). Cuando la suerte está echada: estudio comparativo de los factores asociados al rendimiento en la psu. Revista electrónica iberoamericana sobre la calidad, eficiencia y cambio en educación, 5, 259-263.

Covadonga, m. (2001). Factores familiares vinculados al bajo rendimiento. Revista complutense de educación, 12 (1),81-113.

Cubares, m. (2014), definición de taller.

Creer. (2020). Cifras sobre la educacion rural en el perú. Lima.

Duran, b. (2004). Desarrollo, felicidad, calidad de vida y bienestar humanos. Breves anotaciones sobre su historia y filosofía. Recuperado de http://webcache.googleusercontent.com/search?q=cache:kasx9ozm5bmj:www.fua c.edu.co/download/humano/boletin_2.doc $+\& \mathrm{~cd}=1 \& \mathrm{hl}=\mathrm{es} \& \mathrm{ct}=\mathrm{c} \operatorname{lnk} \& \mathrm{gl}=\mathrm{pe}$

Durrant, joan. 2008. Manual sobre disciplina positiva. Santiago de chile: asociación chilena pro naciones unidas (achnu). 
Edel, r. (2003). El rendimiento académico: concepto, investigación y desarrollo. Reice revista electrónica iberoamericana sobre calidad, eficacia y cambio en educación, 1(2). Recuperado de http://goo.gl/qy3d90

Egg, a. (1999), el taller como sistema de la enseñanza y aprendizaje.

Erazo, o. (julio de 2018). Programa de hábitos escolares para mejorar el bajo rendimiento académico en estudiantes de bachillerato de un colegio público de popayáncolombia. Obtenido de scielo: http://www.scielo.org.co/scielo.php?script=sci_arttext\&pid=s 1692$58582018000200117 \&$ lang=es.

Escalante,h. (2018) bienestar psicológico y rendimiento académico de los estudiantes de primer año de formación inicial docente del instituto pedagógico nacional monterrico. Lima-perú.

Espinoza, e \& calva, d. (2020). La ética en las investigaciones educativas. Revista universidad y sociedad, 12(4), 333-340. Epub 02 de agosto de 2020. Recuperado en 11 de julio de 2021, de http://scielo.sld.cu/scielo.php?script=sci_arttext\&pid=s2218$36202020000400333 \& \operatorname{lng}=\mathrm{es} \&$ tlng=es.

Esquivel, j. Y venegas, v. (2013). Preparación de la tesis universitaria. Juan gutemberg editores impresores e.i.r.l.

Stenhouse, 1. Y. (1975). La disciplina en la escuela. Argentina: el ateneo.

Estévez, c. Y huescar, e. (2009). Conducta prosocial y rendimiento académico en estudiantes españoles de eso. Anales de psicología, 25, 93-101.

Estrada, m. (2019) en su investigación denominada "influencia del clima familiar en el rendimiento escolar del área personal, tumbes - perú. 2019"

Fajardo, f; maestre, m; et al (2017) en su tesis titulada "análisis del rendimiento académico de los alumnos de educación secundaria obligatoria según las variables familiares educación xxi, vol. 20, núm. 1, 2017, pp. 209-232 universidad nacional de educación a distancia madrid, españa.

Fernández, r. Y muñiz, m. (2012). Colegios concertados y selección de escuela en españa: un círculo vicioso. Presupuesto y gasto público, 67, 97-118 
Figueroa, 1., \& chumpitaz, j. (2013). Implementacion de un blog educativo para el mejoramiento del rendimiento academico en mattematicas de estudiantes del segundo grado de secundaria de la ie 6048-villa el salvador. Lima.

Garbanzo, v. (2007). Factores asociados al rendimiento académico en los estudiantes universitarios, una reflexión desde la calidad de la educación superior pública.

García,d.(2010) «estudio sobre la asertividad y habilidades sociales en el alumnado de educación social.» revista de educación (12): 7-22.

Garbanzo, v. (2007). Factores asociados al rendimiento académico en los estudiantes universitarios, una reflexión desde la calidad de la educación superior pública.

Garcés, d. (2012). Incidencia de los rasgos de personalidad en las conductas agresivas de los integrantes de las barras «club macará» temporada 2010/2011. [tesis doctoral]. Universidad técnica de ambato. Recuperado de http://goo.gl/zhxiwr.

Garreta, j. (ed). (2007). La relación familia-escuela. Lleida: editions de la universidad de lleida.

Gil flores, j. (2011). Medición del nivel socioeconómico familiar en el alumnado de educación primaria. Revista de educación. 362, 298-322.

Gómez,m,et, al gracia s.(1993). Propuestas de intervención en el aula: técnicas para lograr un clima favorable en clase. Madrid: narcea.

Jaramillo, 1. (2010) pedagogía y didáctica general. Quito: universidad tecnológica equinoccial.

Jiménez, m. (2019) disciplina positiva y la modulación del comportamiento de estudiantes de educación general básica en el ecuador. Quito.

Makarenko y arellano, c. (2002). En la escuela y la disciplina.

Martínez, 1. (2002). La obediencia de los hijos entre los seis y los 12 años. (2002). Ministerio de educación (2004). "módulo odae: promoviendo el proceso de enseñanza - aprendizaje". Lima - perú, editorial del ministerio de educación

Maslow, a. (1999) las jerarquías de necesidades humanas.

Mella, o. Y ortiz, i. (1999). Rendimiento escolar. Influencias diferenciales de factores externos e internos. Revista latinoamericana de estudios educativos,1, 69-92.

Mesa de concertacion para la lucha contra la pobreza tumbes. (2019). Situacion de las niñas, niños y adolescentes de la region tumbes. Tumbes.

Ministerio de educacion. (2017). Peru ¿como vamos en educacion? Lima. 
Ocampos, r. (2018). Efectos de un programa de inteligencia emocional en el rendimiento esxcolar en la ie inmaculada concepcion tumbes 2018. Tumbes.

Ocde. (02 de febrero de 2016). Ocde publica estudio de rendimiento escolar. Obtenido de https://www.dw.com/es/ocde-publica-estudio-de-rendimiento-escolar/a19037188.

Ocde (2009) pisa programa para la evaluación internacional de losalumnos ocde. Informe español. Ministerio de educación. Recuperado dehttp://goo.gl/wa92xd

Osorio, j., \& castiblanco, 1. (2019). Efectividad del b-learning sobre rendimiento académico y retención en estudiantes en educación a distancia. Scielo, 212 -223.

Pineda, m. (2007). Concepto de rendimiento académico. Recuperado de 87 http://www.psicopedagogia.com/definicion/rendimiento\%20academico

Possitive discipline association. 2017. Positive discipline. 23 de enero. Último acceso: 4 de diciembre de 2017. Https://www.positivediscipline.org/2017

Pronabec. (2013). El alto rendimiento escolar para beca 18. Lima.

Quinteros, d. (2013). Efectos de la aplicación de un programa de bienestar psicológico en estudiantes universitarios de una universidad privada. (tesis inédita de doctorado). Universidad femenina del sagrado corazón, lima.

Rojas, 1 [2017]. Investigación e innovación metodológica. Obtenido de http://investigacionmetodologicaderojas.blogspot.com/2017/09/poblacion-ymuestra.html

Sánchez, 1. (1993) tesis “disciplina escolar”.

Sepúlveda, j. M. (2012). El manejo de los comportamientos disruptivos en el aula de educación primaria”. España: universidad de valladolid.

Sureda, r. (2003). La disciplina en el aula". Argentina: editorial lexus.

Tejedor, f. J. (2003). Poder explicativo de algunos determinantes del rendimiento en los estudios universitarios. Revista española de pedagogía, 61, 5-32.

Torres, a. (2017). Programa de habibilades para mejorar el rendimiento academico del area de ingles en los estudiantes de iesp-david sanchez infante. Trujillo.

Unicef ecuador. S.f. El acoso escolar, otra forma de violencia. Último acceso: 22 de mayo de 2018. Https://unicef.org.ec/acoso-escolar-otra-forma-violencia/. 
Valdés, e. Pujol, 1. (2012). Autorregulación y rendimiento académico en la transición secundaria-universidad. Revista latinoamericana de ciencias sociales, niñez y juventud, 10(1), 367-378.

Vásquez, d \& villa, j (2018), titulada "la disciplina escolar y el aprendizaje del área personal social en los estudiantes del quinto grado de educación primaria de la institución educativa manuel gonzales prada de huaycán, ugel nº6-dre de lima metropolitana" 2018.

Watenburger, s. (1994). Parenting. Discipline and guidance. (crianza de los hijos. La disciplina y orientación)

Weiner, b. (1974) características de éxitos o fracasos en la educación.

White, k. R. (1982). The relation between socioeconomic status and academic achievement. Psychological bulletin, 91, 461-481.

Yerco. (2017). Tus clases. Obtenido de tus clases: https://www.tusclases.pe/blog/2017/2/problemas-conducta-relacion-rendimientoninos-comienzan-estudiantes.

Zamudio, r. (2010) disciplina escolar: desarrollo y aplicación de un programa actitudinal cognitivo para la formación permanente del profesorado de educación primaria. Barcelona: universidad autónoma de Barcelona. 